\title{
Caracterizacion de las Ramas del Arco Aórtico en una Muestra de Poblacion Colombiana. Un Estudio con Material de Autopsia
}

\author{
Characterization of Aortic Arch Branches in a Colombian Population Sample. \\ A Study with Autopsy Material \\ "Néstor Eduardo Herrera Ortiz; **Luis Ernesto Ballesteros Acuña \& ${ }^{* * * *}$ Pedro Luis Forero Porras
}

HERRERA, N. E.; BALleSteros, L. E. \& FORERO, P. L. Caracterizacion de las ramas del arco aórtico en una muestra de población colombiana. Un estudio con material de autopsia. Int. J. Morphol., 30(1):49-55, 2012.

RESUMEN: La variabilidad del arco aórtico y sus ramas emergentes presentan implicaciones en los abordajes quirúrgicos de tórax y cuello, y posiblemente en el desarrollo de procesos ateromatosos ubicados a ese nivel y accidentes cerebrovasculares. Se evaluaron 122 arcos aórticos de individuos adultos de ambos sexos obtenidos como material de autopsia. Se identificó la configuración general de la emergencia de las ramas colaterales de los arcos y se determinó la morfometría de sus componentes con medición electrónica. Se observó la presencia de la configuración usual (tipo A) en 87 arcos (71,3\%); un tronco braqui-bicarotideo (tipo B) en 21 piezas anatómicas $(17,2 \%)$ y en 10 casos $(8,2 \%)$ la arteria vertebral izquierda se originó directamente del arco aórtico (tipo C); en 4 especímenes (3,3\%) se presentó emergencia atípica de las ramas. El calibre de la aorta en el punto previo a la emergencia de sus ramas y justo después de emitir su última colateral fue de 20,1 $\mathrm{mm}$ (DE 3,19) y 17,2 $\mathrm{mm}$ (DE 2,57) respectivamente, con una disminución del 14,5\%. El calibre de las arterias sublavias $(7,7 \mathrm{~mm}, \mathrm{DE} 1,10)$ fue significativamente mayor $(\mathrm{P}=0,0001)$ que el de las arterias carótidas $(6,4 \mathrm{~mm}, \mathrm{DE} 0,78)$. El diámetro de las arterias carótidas derecha e izquierda fue de 6,5mm (DE 0,81 ) y 6,3 mm (DE 0,75) respectivamente. La arteria subclavia derecha presentó mayor calibre que la izquierda $(7,9 \mathrm{~mm}$, DE 1,09; 7,6mm, DE 1,12) sin diferencia estadisticamente significativa $(\mathrm{P}=0,0801)$. La distancia entre el origen del tronco braquiocefálico y el de la subclavia izquierda fue de $32,8 \mathrm{~mm}(\mathrm{DE} 6,16)$ y la longitud del tronco braquiocefálico fue $30,2 \mathrm{~mm} \pm 5,27$. Se destaca la alta frecuencia de arcos con emergencia de dos y cuatro ramas. Los calibres de las ramas son menores a lo reportado en la literatura.

PALABRAS CLAVE: Arco aórtico; Tronco braquiocefálico; Arteria carótida; Arteria subclavia; Arteria vertebral.

\section{INTRODUCCIÓN}

El arco aórtico (AA), ubicado en el mediastino superior, usualmente emite el tronco braquiocefálico (TBC), la arteria carótida común izquierda (ACCI) y la subclavia izquierda (ASI), que participan en la irrigación de la cabeza y los miembros superiores (Moore \& Dalley, 2007; Draken et al., 2010). De la cuarta a la octava semana del desarrollo embrionario los factores que actúan sobre reguladores genéticos determinan modificaciones a nivel del tercer y cuarto arcos aórticos, y la consecuente distribución final variante de las ramas que se derivan de esas estructuras (Momma et al., 1999; Sadler, 2004; Bathia et al., 2005). La principal variabilidad observada en la emergencia de las ramas del AA se caracteriza por la presencia de dos y cuatro ramas observada entre el 6-32\% de los casos (Wright, 1969; Zamir \& Sinclair, 1990; Satyapal et al., 2003; Berko et al.,
2009; Alsaif \& Ramadan, 2010; Jakanani \& Adair, 2010; Müller et al., 2011).

Los AA de dos ramas usualmente se configuran por la presencia del tronco braquiobicarotideo ( $\mathrm{TBbC}$ ), formado de la unión del TBC con la ACCI, y de la ASI. En los arcos con 4 ramas, con mayor frecuencia se observa que la rama adicional es la arteria vertebral izquierda (AVI) que emerge entre la carótida y subclavia izquierda (Matula et al., 1997; Komiyama et al., 2001; Yamaki et al., 2006; Berko et $a l)$.

La importancia del conocimiento de las variaciones vasculares, de manera particular del AA y sus ramas radica, además del concepto académico, en su impacto sobre los

* Médico Universidad Industrial de Santander, Bucaramanga, Colombia.

** Profesor Titular Departamento de Ciencias Básicas, Universidad Industrial de Santander, Bucaramanga, Colombia.

**** Profesor Asistente Departamento de Ciencias Básicas, Universidad Industrial de Santander, Bucaramanga, Colombia. 
procedimientos hemodinámicos, en la etiología de las lesiones cerebrales derivadas de la manipulación de estos vasos y en el manejo del trauma torácico superior y del cuello. El desconocimiento de las expresiones variantes de las ramas derivadas del AA puede precipitar la ocurrencia de iatrogenias durante los procedimientos quirúrgicos (Matula et al.; Satyapal et al.; Nayak et al., 2006; Jakanani \& Adair).

La evaluación de la emergencia de las ramas del AA ha sido sido realizada en grupos poblacionales variados y a través de métodos anatómicos convencionales e imagenológicos (De Garis et al., 1933; Grande et al., 1995; Satyapal et al.; Bathia et al.; Yamaki et al.; Berko et al.; Natsis et al., 2009; Bhattarai \& Poudel, 2010; Ogeng'o et al., 2010; Alsaif \& Ramadan). Consideramos importante una caracterización morfológica de estas estructuras, desarrollada en material cadavérico fresco, que contemple población colombiana y que contribuya a la obtención de una casuística propia de referencia, al mejoramiento de la interpretación de sus imágenes y al manejo de eventos quirúrgicos.

\section{MATERIAL Y MÉTODO}

En este estudio no probabilístico y descriptivo transversal se evaluaron los AA y sus ramas de 122 cadáveres frescos de sexo masculino, sin signos de patología o trauma toraco-cervical, a quienes se les practicó necropsia en el laboratorio de Patología de la Universidad Industrial de Santander o en el Instituto de Medicina legal y de Ciencias Forenses de Bucaramanga-Colombia.

El segmento vascular comprendía desde la emergencia cardiaca de la arteria aorta hasta su segmento descendente y en el caso de las colaterales hasta el nivel del tercio inferior del cuello y cara superior de la clavícula. Los especímenes fueron manipulados en fresco, y su disección condujo a la liberación del tejido conectivo adyacente.

Se registraron las diversas formas de emergencia de las ramas del AA y se agruparon de acuerdo a los patrones establecidos por Adachi (1928). Luego, se practicó incisión longitudinal por la cara posterior de los AA y de sus ramas.

Seguidamente se procedió a medir con calibrador digital Mitotuyo® los perímetros de las ramas a $0,5 \mathrm{~cm}$ de su origen, y el de la aorta antes de la emergencia de la primera rama y después del origen de la ultima rama emergente. Mediante aplicación de la fórmula Perímetro $=2 \mathrm{r}^{*} \pi$ se determinó los diámetros de las estructuras vasculares. Igualmente, se hizo medición de las distancias entre los orígenes de las colaterales.

De cada una de las piezas evaluadas se obtuvieron registros fotográficos. Se diseñó una base de datos en Excel. Los análisis estadísticos se realizaron en STATA 8.0. En el análisis de los datos las variables continuas fueron descritas con sus promedios y desviación estándar, igualmente las variables nominales con sus proporciones. Se realizaron las pruebas estadísticas de chi $\left(\mathrm{X}^{2}\right)$ cuadrado y test de student aceptando un error alfa hasta de un 5\%.

\section{RESULTADOS}

Se evaluaron 122 AA obtenidos de especímenes cadavéricos masculinos con edad promedio de 32,4 años (DE 15,26). Se observó el patrón usual de 3 ramificaciones (tipo A) (Fig. 1) en 87 casos (71,3\%); $\mathrm{El}$ arco tipo $\mathrm{B}$, con la emergencia de 2 ramas, un TBbC y la ASI se presentó en 21 casos (17,2\%) (Fig. 2), mientras que en 10 especímenes $(8,2 \%)$ se observó arcos de 4 ramas con la emergencia de la AVI directamente del arco aórtico entre las arterias carótida común y subclavia izquierda (tipo C) (Fig. 3). En 4 especímenes (3,3\%) se identificaron diversas expresiones en el numero y ubicación de las ramas, prevaleciendo en todas la emergencia de la AVI desde el arco aórtico.

En un caso $(0,8 \%)$ se observó el AA con emergencia de tres ramas, con origen del TBbC, ACCI y la AVI. (tipo D) (Fig. 4). Una

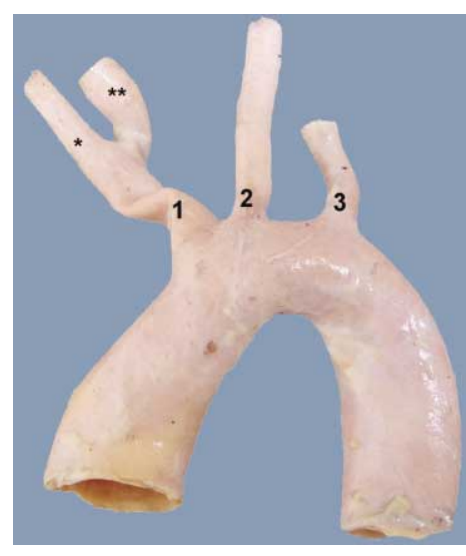

Fig. 1. Arco aórtico tipo A. 1.Tronco braquicefalico del cual emerge la arteria subclavia derecha (1) y la arteria carotida común derecha (11); 2. Arteria carótida izquierda; 3 . Arteria subclavia izquierda.

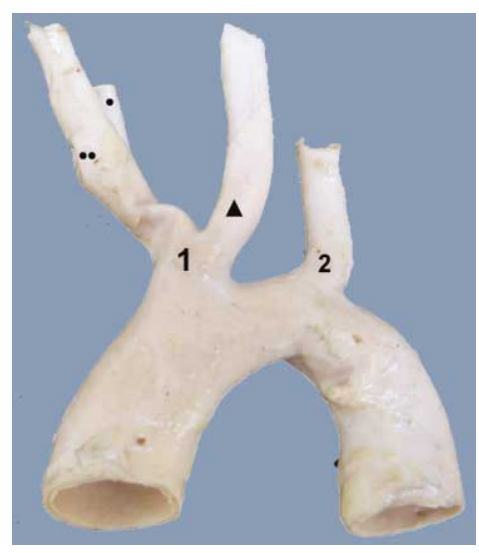

Fig. 2. Arco aórtico tipo B. 1. Tronco braquio-bicarotideo del que se desprende la arteria carótida común izquierda (s), arteria carótida común derecha (1) y la arteria subclavia derecha (11); 2. Arteria subclavia izquierda. 


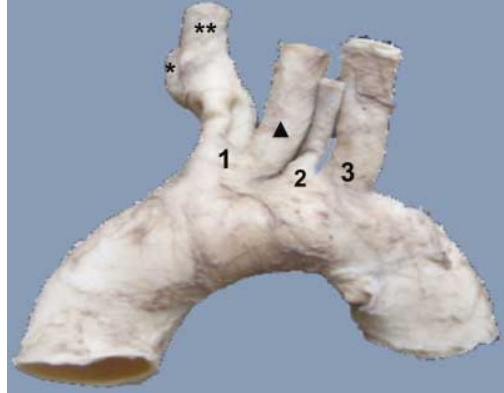

Fig. 3. Arco aórtico tipo C. 1. Tronco braquiocefálico; 2. Arteria carótida común izquierda; 3. Arteria vertebral izquierda; 4. Arteria subclavia izquierda.

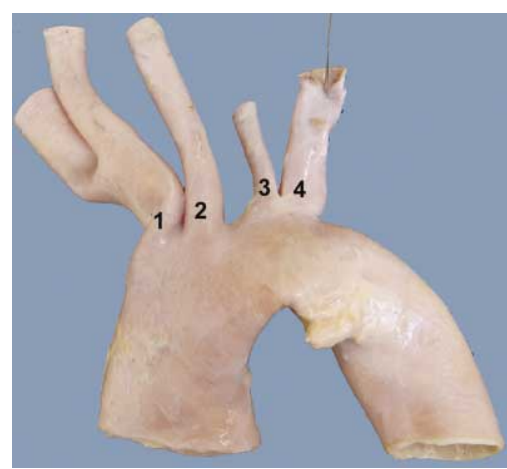

Fig. 4. Arco aortico tipo D. 1. Tronco braquio-bicarotideo del que se desprende la arteria carótida común izquierda (s), arteria carótida común derecha (11) y la arteria subclavia derecha (1);2. Arteria vertebral izquierda; 3 . Arteria subclavia izquierda.

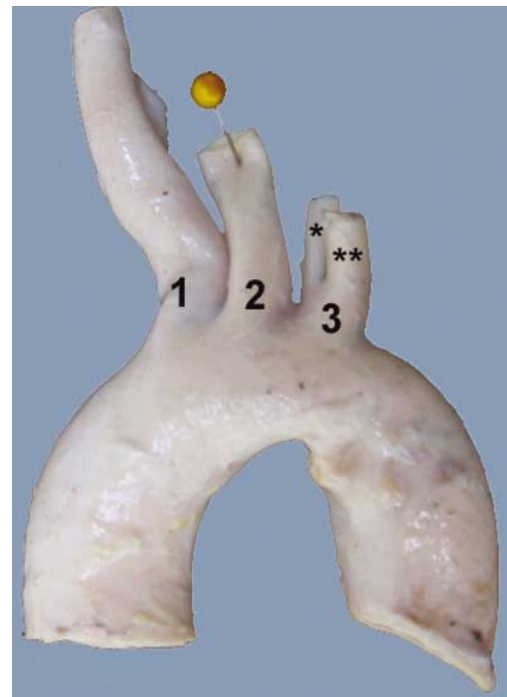

Fig. 5. Arco aórtico tipo E. 1. Tronco braquicefalico; 2 . Arteria carotida común izquierda; 3 . Arteria vertebral izquierda (1) y arteria subclavia izquierda (11) originadas de un tronco común. muestra $(0,8 \%)$ presentó arco de tres ramas, con origen común de la arteria subclavia izquierda y arteria vertebral homónima, formando un mismo "ostium" (tipo E) (Fig. 5). En un espécimen $(0,8 \%)$ del arco aórtico emergieron cinco ramas: arteria carótida común derecha (ACCD), ACCI, AVI, ASI por su convexidad superior y la Arteria subclavia derecha (ASD) aberrante por su cara posterior (tipo F) (Fig. 6). Adicionalmente, se observó un arco de seis ramas, con presentación similar al ante-

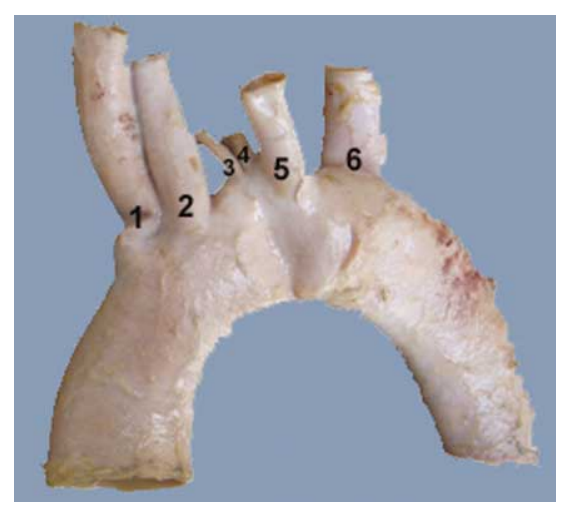

Fig. 6. Arco aórtico tipo F. 1. Arteria carotida común derecha; 2 . Arteria carotida común izquierda; 3 . Arteria vertebral izquierda; 4. Arteria subclavia izquierda; 5 Arteria subclavia derecha aberrante.

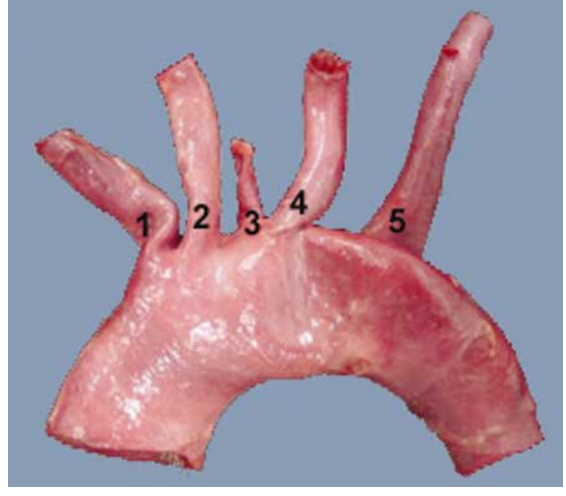

Fig. 7. Arco aórtico tipo G. 1. Arteria carotida común derecha; 2. Arteria carótida común izquierda; 3. Arteria tiroidea; 4. Arteria vertebral izquierda; 5 . Arteria subclavia izquierda; 6. Arteria subclavia derecha. rior, pero con emergencia de la arteria tiroidea inferior, adyacente al origen de la AVI (tipo G) (Fig. 7).

Los calibres de la aorta medidos antes y después de la emergencia de las ramas fueron $20,1 \mathrm{~mm}(\mathrm{DE} 3,19)$ y $17,2 \mathrm{~mm}(\mathrm{DE} 2,57)$ respectivamente, presentándose una reducción del calibre del 14,5\%. El calibre de las arterias subclavias fue 7,7mm (DE $1,1)$, significativamente mayor $(\mathrm{P}=0,0001)$ que el de las arterias carótidas $(6,4 \mathrm{~mm}$, DE 0,78). La ASD presentó mayor calibre que la ASI $(7,9 \mathrm{~mm}, \mathrm{DE} 1,09 ; 7,6 \mathrm{~mm}, \mathrm{DE}$ $1,12)$ sin diferencia estadísticamente significativa $(\mathrm{P}=0,0801)$. Igualmente, la $\mathrm{ACD}$ presentó mayor calibre que la izquierda $(6,5 \mathrm{~mm}, \mathrm{DE} 0,81 ; 6,3 \mathrm{~mm}$, DE 0,75$)$ $(\mathrm{P}=0,0671)$.

En relación con el lado de presentación, el calibre de la ASD y ACCD fue mayor en $68(55,7 \%)$ especimenes; en 8 casos $(6,6 \%)$ el calibre fue igual o similar y en el $37,7 \%$ las ramas del lado izquierdo presentaron mayor calibre. El calibre del TBbC fue de 14,3 mm (DE 2.14). El TBC presentó una longitud de 30,2mm (DE 5,27 ) en un rango de 19,4 a $42,4 \mathrm{~mm}$ y un calibre de $9,7 \mathrm{~mm}$ (DE 1,61).

La longitud del segmento del AA en el que se originan sus ramas (medido entre los bordes laterales del TBC y de la ASI) fue de $32,77 \mathrm{~mm} \pm 6,16$, con un rango de 21,1 a 49,6mm; la mayor distancia entre los origenes de las diversas ramas emergentes del arco fue entre la ACI y la ASI con 5,3mm (DE 3,72), la cual fue significativamnte mayor $(\mathrm{P}=0.0001)$ que la observada entre el TBC y la ACI $(2,23 \mathrm{~mm}, \mathrm{DE} 0,91)$; igualmente, las distancias entre los origenes de las otras ramas en cada uno de los diversos tipos fueron menores (Tabla I).

Las AVI originadas directamente de los AA presentaton un calibre promedio de $3,9 \mathrm{~mm}$ (DE 1,16), de las cuales $4(28,6 \%)$ presentaron un calibre menor a $3 \mathrm{~mm}$ 
Tabla I. Distancias entre los orígenes de las ramas emergentes de 122 arcos aórticos de individuos colombianos en relación con los tipos de expresión. ATB-ASI: Entre tronco braquiocefálico y subclavia izquierda; ATB-ACI: Entre tronco braquiocefálico y carótida izquierda; ACI-ASI: Entre carótida izquierda y subclavia izquierda; ACI-AVI: Entre carótida izquierda y vertebral izquierda; AVI-ASI: Entre vertebral izquierda y subclavia izquierda.

\begin{tabular}{|c|c|c|c|c|c|}
\hline DISTANCIA en mm y DS $( \pm)$. & ATB - ASI & ATB - ACI & ACI - ASI & ACI - AVI & AVI - ASI \\
\hline Tipo A & $33,01 \pm 6,43$ & $2,27 \pm 0,94$ & $5.35 \pm 3,78$ & & \\
\hline Tipo B & $31,55 \pm 5,16$ & & & & \\
\hline Tipo C & $34,37 \pm 6,82$ & $1,97 \pm 0,57$ & & $2.87 \pm 2,32$ & $1.75 \pm 0,64$ \\
\hline Otros Tipos & $27,89 \pm 3,35$ & $2,24 \pm 0,90$ & $3,3 \pm 2,21$ & 2.44 & $2,40 \pm 0,82$ \\
\hline TOTAL MUESTRA & $32,77 \pm 6,16$ & $2,23 \pm 0,91$ & $5,3 \pm 3,72$ & $\mathbf{2 , 8 3} \pm 2,32$ & $1,90 \pm 0,68$ \\
\hline
\end{tabular}

DISCUSIÓN

La expresión morfológica de las ramas emergentes del AA ha sido descrita en estudios realizados en diversos grupos poblacionales con resultados que presentan un amplio espectro de variabilidad. El tipo usual de tres ramas con un TBC, ACI y ASI, ha sido reportada con una incidencia mayor al 85\%, por Nelson \& Sparks (2001) (94,3\%), Wright (85\%), Nayak et al. (91,4\%), Müller et al. (86.7\%) y Satyapal et al. (94,7\%). Otros autores (Adachi; De Garis et al.; Natsis et al.; Grande et al.; Shin et al., 2008; Bhattarai \& Poudel; Alsaif \& Ramadan; Gupta \& Sodhi, 2005; Zamir \& Sinclair) han informado frecuencias medias, en un rango de 75 a $84 \%$. En nuestro estudio se observó este tipo A en el 71,3\% de los casos evaluados, resultados que son concordantes con los trabajos de Berko et al. (65,9\%), Ogeng'o et al. (67,3\%); Bathia et al. (74,1\%\%) y Jakanani \& Adair (74\%) ubicándose en el grupo que reporta una relativa baja incidencia de esta forma usual, en un rango de 65 a 74\%. Una incidencia muy baja del tipo A ha sido reportada por De Garis et al. en muestra de negros americanos (47,9\%), Rojas \& Ballesteros (2009) (56,9\%) y Yeri et al. (2011) (57\%), trabajos en los que las formas variantes, especialmente los tipos B y C presentan un alto porcentaje.

En nuestro estudio, el tipo B caracterizado por la emergencia desde el AA de un TBbC y de la ASI, presentó una frecuencia de 17,2\% resultado que es concordante con la mayoría de trabajos previos (Adachi; Alsaif \& Ramadan; Bhattarai \& Poudel; Natsis et al.; Gupta \& Sodhi; Grande et al.; Jakanani \& Adair; Zamir \& Sinclair) que reportan este rasgo anatómico en un rango del 10 a 20\%. Una alta frecuencia de este tipo es reportada por Berko et al. (27,4\%), De Garis et al. en población negra (35,8\%), Rojas \& Ballesteros $(25,5 \%)$, Ogeng'o et al. $(25,7 \%)$ y Yeri et al. $(36,4 \%)$, mientras que frecuencias bajas con cifras menores al $10 \%$ son reportadas por Shin et al., Müller et al., Satyapal et al. y Wright. Para destacar, Nelson \& Sparks en una muestra de 193 especímenes de origen japonés solo reportó este rasgo en el $1,1 \%$.
En el arco de 4 ramas, tipo C, la arteria vertebral emerge de la superficie dorsal de la aorta entre la ACI y la ASI es reportado por diversos autores (Adachi; Alsaif \& Ramadan; Berko et al.; De Garis et al.; Matula et al.; Grande et al.; Jakanani \& Adair; Komiyama et al.; Müller et al.; Nelson \& Sparks; Ogeng'o et al.; Yamaki et al.; Yeri et al.; Zamir \& Sinclair) con una frecuencia entre 0,8-6\%. En nuestro estudio se observó con una frecuencia ligeramente mayor $(8,2 \%)$, similar a lo reportado por Wright et al. y Shin et al.; mientras que la mayor incidencia $(13,9-15,7 \%)$ corresponde a los trabajos de Bathia et al. y Rojas \& Ballesteros realizados en muestras de población australiana y colombiana.

Los tipos D, E, F y G observados en nuestra serie con una frecuencia global de $3,3 \%$ no son reportados, lo que confirma su escasa presentación (Bhattarai \& Poudel; Gupta \& Sodhi; Grande et al.; Jakanani \& Adair; Shin et al.; Zamir \& Sinclair). Estas expresiones del AA han sido descritas también con un baja incidencia, en un rango de 0,4-2,8\% (De Garis et al.; Müller et al.; Natsis et al.; Nayak et al.; Satyapal et al.).

Del amplio espectro en la incidencia de las expresiones variantes de las ramas emergentes del AA, reportadas en diversos grupos poblacionales, señalada desde 1,1\% (Nelson et al.) hasta 36\% (De Garis et al.; Yeri et al.) para los arcos de dos ramas y desde $0,8 \%$ (Natsis et al.) hasta $15,7 \%$ (Rojas \& Ballesteros) para los arcos de 4 ramas, puede inferirse que además de las diferentes configuraciones genéticas propias de cada grupo étnico, debe considerarse el tamaño de las muestras evaluadas como elemento determinante; es posible que en estudios con muestras pequeñas (Grande et al.; Shin et al.; Rojas \& Ballesteros; Yeri et al.) se den sesgos a favor o en contra de ciertos tipos de expresión, los cuales pueden ser homogenizados en parte, si el universo de la muestras fuera mayor a cien especímenes. 
Con relación a los calibres del AA evaluados antes y después de la emergencia de sus ramas, los resultados de nuestra serie $(20,1 \mathrm{~mm}$ y $17,2 \mathrm{~mm}$ respectivamente) son menores a los de Wright et al., Grande et al. y Yeri et al., quiénes repor$\tan 23 \mathrm{~mm}$ y 18,2 a $19,5 \mathrm{~mm}$ respectivamente, con una reducción de su calibre entre los puntos señalados del 15-21\%, mientras que en nuestro estudio esta reducción fue de 14,5\%. En concordancia con estos autores, el calibre de las arterias subclavias es significativamente mayor que el de las carótidas; igualmente, las arterias subclavia y carótida derechas presentan un calibre ligeramente mayor que las del lado izquierdo.

Los calibres de cada una de las ramas evaluadas en nuestro trabajo son menores a los reportes previos (Tabla II). Esta diferencia podría ser explicada por la utilización por parte de los autores de diversas técnicas de medición y por la menor talla de los sujetos del grupo poblacional colombiano. El conocimiento de los calibres de estos vasos son de ayuda para seleccionar el tamaño apropiado y las características de los de los catéteres que se usen en los procedimientos endovasculares que involucren estas estructuras (Shin et al.).

Se presenta concordancia con Wright y Shin et al. en torno a la distancia entre el origen del TBC y la ASI $(32,7 \mathrm{~mm})$, en tanto que Wright reporta una distancia de $7 \mathrm{~mm}$ entre el origen de la ACCI y la ASI, cifra que es considerablemente mayor a lo hallado en nuestro estudio. Teniendo en cuenta la poca información disponible en torno a las distancias entre las ramas emergentes del AA se requiere considerar este tópico en futuras evaluaciones que se realicen en especímenes adultos.

Las variaciones de las ramas emergentes del AA están determinadas por selección del gen q11 del cromosoma 22, el cual desencadena alteraciones en los complejos procesos embriológicos de regresión, absorción y retención de estos vasos (Momma et al.). El Origen de la AVI desde la superficie dorsal del AA sugiere que parte del arco proviene de la sépti- ma arteria intersegmentaria izquierda o a una mayor reabsorción del tejido embrionario de la ASI entre el origen del AA y la AVI (Bathia et al.). Igualmente, TBbC, en el Arco de 2 ramas, se expresa en las primeras fases del desarrollo embrionario como estructuras separadas (TBC y ACCI) que cada vez más se acercan una a la otra. Es probable que la fusión entre las dos ramas sea el resultado de una amplia migración de estas (Grande et al.; Ogeng'o et al.).

La ASD aberrante se destaca entre las expresiones raras de las ramas emergentes del AA (Fig. 6). Esta variación es resultado de la interrupción en el desarrollo embriológico del cuarto arco aórtico derecho entre la carótida y las arterias subclavias. Nuestro hallazgo $(1,6 \%)$ es concordante con estudios previos (De Garis et al.; Liechty et al., 1957; Berko et $a l$.) que la reportan en un rango de $0,4-2 \%$. Su curso aberrante suele ser retro esofágico y de evolución asintomática, descubriéndose como un hallazgo incidental. Dadas sus importantes relaciones topográficas, es importante su reconocimiento previo en el planeamiento de procedimientos quirúrgicos de esófago y de las estructuras contenidas en el mediastino superior.

Las expresiones variantes de las ramas del AA han sido asociadas con la formación de placas de ateromas a ese nivel debido probablemente a modificaciones del flujo sanguíneo que determina lesión del endotelio. Esto es particularmente importante en razón a que a partir de las placas se pueden formar émbolos que se constituyen en alto riesgo de enfermedades cerebro vasculares (Rodkiewicz et al., 1985; Amarenco et al., 1992).

Se señala una mayor incidencia estadísticamente significativa de disección de la pared vascular de la AVI que causa isquemia cerebral cuando se origina directamente del AA, con relación a la que se presenta del lado derecho (Yamaura et al., 1990). Esto puede explicarse por defecto congénito estructural de la pared y las alteraciones del flujo cerebral pul-

Tabla II. Calibres en mm de las ramas emergentes del arco aórtico reportados en diversos estudios. TBbC: tronco braquiobicarotideo; TBC: Tronco braquicefálico; ACCD: Arteria carotida común derecha; ASD: Arteria subclavia derecha; ACCI: Arteria carótida común izquierda; ASI: Arteria subclavia izquierda.

\begin{tabular}{lcccccc}
\hline Autor & TBbC & TBC & ACCD & ASD & ACCI & ASI \\
\hline Wright,1969 & 14,5 & 12,5 & - & - & 7.5 & 10 \\
Grande et al., 1995 & - & 11,2 & - & - & 8,5 & 8,8 \\
Matula et al.,1997 & - & - & 8,8 & 12,3 & 8,7 & 9,9 \\
Nelson \& Sparks, 2001 & - & - & 8,9 & 10,9 & 8,8 & 10,7 \\
Sora et al., 2002 & 18,7 & - & 9,6 & 11,1 & 9,6 & 11,2 \\
Shin et al,, 2008 & 18,3 & - & - & - & 9,8 & 10,6 \\
Yeri et al., 2011 & 17,6 & 11,5 & - & - & 7,9 & 10,1 \\
Presente estudio & 14,3 & 9,7 & 6,5 & 7,9 & 6,3 & 7,6 \\
\hline
\end{tabular}


sátil, observado en la vertebral que emerge de la aorta y que origina daño en el endotelio a diferencia del flujo amortiguado que se presenta cuando se deriva de la subclavia (Bernardi \& Dettori, 1975; Komiyama et al.).

Las complicaciones de angiografía cerebral temporales o permanentes son de $1-2 \%$ y se deben a una pobre localización de las ramas y a su angulación, lo que determina dificultad en el avance de los catéteres, lo cual puede desencadenar embolismo desde los ateromas ubicados en las ramas de la aorta (Willinsky et al., 2003; Kim \& Suh, 2006). Estas complicaciones podrían reducirse al tener en cuenta las variaciones de las emergentes de la aorta en pacientes viejos con enfermedad cardiovascular.

El estudio de las ramas emergentes del AA en especímenes cadavéricos ha generado gran información sobre la configuración de estas estructuras, pero con la limitante de no aportar sobre sus dinámicas vasculares, aspectos que son suministrados de manera puntual por la resonancia magnética angiográfica; por lo que se hace imperativo la utilización de estos últimos recursos en el planeamiento de procedimientos quirúrgicos que comprometan estos vasos. Además, en las intervenciones de urgencias es indispensable que el cirujano de tórax posea un conocimiento amplio de las variaciones que comprometen a estas ramas, que sumada a una cuidadosa exanimación y correcta identificación de estos vasos, pueden evitar complicaciones muchas veces fatales durante estos procedimientos (Matula et al.; Willinsky et al.; Satyapal et al).

AGRADECIMIENTOS. Al Instituto de Medicina Legal y Ciencias Forenses de Bucaramanga-Colombia y al Laboratorio de Patología de la Universidad Industrial de Santander por el suministro de las muestras requeridas para esta investigación.

HERRERA, N. E.; BALLESTEROS, L. E. \& FORERO, P. L. Characterization of aortic arch branches in a Colombian population sample. A study with autopsy material. Int. J. Morphol., 30(1):49-55, 2012.

SUMMARY: The variability of the aortic arch and its emergent branches have implications in the surgical approaches of the thorax and neck, and possibly in the development of the atheromatous processes located at that level and the cerebrovascular accidents. We evaluated 122 aortic arches from adult individuals of both sexes obtained as autopsy material. We identified the general configuration of the emergence of the collateral branches of the arcs and determined the morphometry of its components with electronic measurement. We observed the usual configuration (type A) in 87 arches (71.3\%); a brachio-bicarotid trunk (type B) in 21 anatomical specimens (17.2\%) and in 10 cases $(8.2 \%)$ the left vertebral artery originated directly from the aortic arch (type C); 4 specimens (3.3\%) presented atypical emergency in the branches. The caliber of the aorta at the point prior to the emergence of its branches and just after casting his last side was $20.1 \mathrm{~mm}$ (DS 3.19) and 17.2mm (DS 2.57) respectively, with a decrease of 14.5\%. The caliber of the subclavian arteries (7.7mm, SD 1.1) was significantly higher $(\mathrm{P}=0.0001)$ than of the carotid arteries $(64 \mathrm{~mm}$, SD 0.78$)$. The diameter of the carotid arteries both right and left were $6.5 \mathrm{~mm}$ (DS 0.81 ) and $6.3 \mathrm{~mm}$ (DS 0.75) respectively. The right subclavian artery presented higher caliber than the left (7.9mm, DS 1.09 ; $7.6 \mathrm{~mm}$, DS 1.12) without significant statistical difference $(\mathrm{P}=0.0801)$. The difference between the origin of the brachiocephalic trunk and the left subclavian artery was 32.8 (DS 6.16); the brachiocephalic trunk length was $30.2 \mathrm{~mm} \pm 5.27$. It highlights the high frequency of arches with emergency of two and four branches. The calibers of the branches are smaller than those reported in the literature.

KEY WORDS: Aortic arch; Brachiocephalic trunk; Carotid artery; Subclavian artery; Vertebral artery.

\section{REFERENCIAS BIBLIOGRÁFICAS}

Adachi, B. Das Arteriensystem der Japaner. Kyoto, KaiserlichJapanischen Universität zu Kyoto, 1928.

Alsaif, H. A. \& Ramadan, W. S. An Anatomical Study of the Aortic Arch Variations. JKAU: Med. Sci., 17(2):37-54, 2010.

Amarenco, P.; Duyckaerts, C.; Tzourio, C.; Bousser, M. G. \& Hauw, J. J. The prevalence of ulcerated plaques in the aortic arch in patients with stroke. N. Engl. J. Med., 326(4):221-5,1992.

Bathia, K.; Ghabriel, M. N. \& Henneberg, M. Anatomical variations in the branches of the human aortic arch: a recent study of a South Australian population. Folia Morphol., 64(3):217-23, 2005.
Bhattarai, C. \& Poudel, P. P. Study on the variation of branching pattern of arch of aorta in Nepalese. Nepal Med. Coll. J., 12(2):84-6, 2010.

Bernardi, L. \& Dettori, P. Angiographic study of a rare anomalous origin of vertebral artery. Neuroradiol., 9:43-7, 1975.

Berko, N. S.; Jain, V. R.; Godelman, A.; Stein, E. G.; Ghosh, S. \& Haramati, L. B. Variants and anomalies of thoracic vasculature on computed tomographic angiography in adults. J. Comput. Assist. Tomogr., 33(4):523-8, 2009.

Draken, R. L.; Vogl, A. W. \& Mitchell, A. W. Gray Anatomy for students. 2nd ed. Philadelphia, Churchill Livingstone, 2010.

De Garis, C.; Black, I. H. \& Riemenschneider, E. Patterns of the aortic arch in American White and Negro stocks, with comparative notes on certain other mammals. J. Anat., 67(Pt 4):599-619, 1933. 
Gupta, M. \& Sodhi, L. Variations in branching pattern, shape,size and relative distances of arteries arising fron arch of aorta. Nepal. Med. Coll. J., 7(1):13-7, 2005.

Grande, N. R.; Costa e Silva, A.; Pereira, A. S. \& Aguas, A. P. Variations in the anatomical organization of the human aortic arch. A study in a Portuguese population. Bull. Assoc. Anat., 79(244):19-22, 1995.

Jakanani, G. C. \& Adair, W. Frequency of variations in aortic arch anatomy depicted on multidetector CT. Clin. Radiol., 65(6):4817, 2010.

Kim, H. J. \& Suh, D. C. Atherosclerotic carotid stenosis: recent update of carotid stenting. Neurointervention, 1:7-17, 2006.

Komiyama, M.; Morikawa, T.; Nakajima, H.; Nishikawa, M. \& Yasui, T. High incidence of arterial dissection associated with left vertebral artery of aortic origin. Neurol. Med. Chir., 41(1):8-11, 2001.

Liechty, J. D.; Shields, T. W. \& Anson, B. J. Variations pertaining to the aortic arches and their branches; with comments on surgically important types. Q. Bull. Northwest Univ. Med. Sch., 31(2):13643, 1957.

Matula, C.; Trattnig, S.; Tschabitscher, M., Day, J. D. \& Koos, W. T. The course of the prevertebral segment of the vertebral artery, anatomy and clinical significance. Surg. Neurol., 48(2):12531,1997 .

Momma, K.; Matsuoka, R. \& Takao, A. Aortic arch anomalies associated with chromosome 22q11 deletion (CATCH 22). Pediatr. Cardiol., 20(2):97-102, 1999.

Moore, K. L. \& Dalley, A. F. Anatomía con orientación clínica. 5a ed. Madrid, Médica Panamericana, 2007.

Müller, M.; Schmitz, B. L.; Pauls, S.; Schick, M.; Röhrer, S.; Kapapa, T. \& Schlötzer, W. Variations of the aortic arch - a study on the most common branching patterns. Acta. Radiol., 52(7):738-42, 2011.

Natsis, K.; Tsitouridis, I. A.; Didagelos, M. V.; Fillipidis, A. A.; Vlasis, K. G. \& Tsikaras, P. D. Anatomical variations in the branches of the human aortic arch in 633 angiographies: clinical significance and literature review. Surg. Radiol. Anat., 31(5):319-23, 2009.

Nayak, S.; Pai, M.; Prabhu, L.; D'Costa, S. \& Shetty, P. Anatomical organization of aortic arch variations in the India: embryological basis and review. J. Vasc. Bras., 5(2):95-100, 2006.

Nelson, M. L. \& Sparks, C. D. Unusual aortic arch variation: distal origin of common carotid arteries. Clin. Anat., 14(1):62-5, 2001.

Ogeng'o, J. A.; Olabu, B. O.; Gatonga, P. M. \& Munguti, J. K. Branching pattern of aortic arch in a Kenyan population. $J$. Morphol. Sci., 27(2):51-5, 2010.
Rodkiewicz, C. M.; Kalita, W.; Kennedy, J. S. \& Pelot, R. On the flow field distortions due to the aortic arch twist. J. Biomech., 18(10):781-7,1985.

Rojas, O. J. D. \& Ballesteros, A. L. E. Branches arise of the aortic arch in human fetus. A descriptive direct study in Colombian population. Int. J. Morphol., 27(4):989-96, 2009.

Sadler, T. Langman Embriología médica con orientación clínica. 9a ed. Buenos Aires, Médica Panamericana, 2004. pp.233-87.

Satyapal, K. S.; Singaram, S.; Partab, P.; Kalideen, J. \& Robbs, J. Aortic arch branch variations - case report and arteriographic analysis. S. Afr. J. Surg., 41(2):48-50, 2003.

Sora, M. C.; Strobl, B.; Förster-Streffleur, S. \& Staykov, D. Aortic arch variation analyzed by using plastination. Clin. Anat., 15(6):379-82, 2002.

Shin, I. Y.; Chung, Y. G.; Shin, W. H.; Im, S. B.; Hwang, S. C. \& Kim, B. A. Morphometric Study on Cadaveric Aortic Arch and Its Major Branches in 25 Korean Adults: The Perspective of Endovascular Surgery. J. Korean Neurosurg. Soc., 44:78-83, 2008.

Willinsky, R. A.; Taylor, S. M.; TerBrugge, K.; Farb, R. I.; Tomlinson, G. \& Montanera, W. Neurologic complications of cerebral angiography: prospective analysis of 2,899 procedures and review of the literature. Radiology, 227(2):522-8, 2003.

Wright, N. L. Dissection study and mensuration of the human aortic arch. J. Anat., 104(Pt 2):377-85, 1969.

Yamaki, K.; Saga, T.; Hirata, T.; Sakaino, M.; Nohno, M.; Kobayashi, S. \& Hirao, T. Anatomical study of the vertebral artery in Japanese adults. Anat. Sci. Int., 81(2):100-6, 2006.

Yamaura, A.; Watanabe, Y. \& Saeki, N. Dissecting aneurysms of the intracranial vertebral artery. J. Neurosurg., 72(2):183-8, 1990.

Yeri, L. A.; Gómez, J.; Fontaneto, S. \& Espósito, M. Variation of the origin of aortic arch branches: in relationship with plates of atheroma. Int. J. Morphol., 29(1):182-6, 2011.

Zamir, M. \& Sinclair, P. Continuum analysis of common branching patterns in the human arch of the aorta. Anat. Embryol., 181(1):31-6, 1990.

\section{Dirección para correspondencia: \\ Dr. Luis Ernesto Ballesteros. \\ Av. González Valencia \# 54-25 (404) \\ Bucaramanga \\ COLOMBIA}

\section{Email: Iballest56@yahoo.es}

Recibido : 27-06-2011

Aceptado: 12-12-2011 